\title{
Dosimetric Comparison Between Carbon-ion Radiotherapy and Photon Radiotherapy for Stage I Esophageal Cancer
}

\author{
YOSUKE TAKAKUSAGI ${ }^{1}$, DAISAKU YOSHIDA ${ }^{1}$, YOHSUKE KUSANO ${ }^{2}$, KIO KANO $^{1}$, WATARU ANNO ${ }^{1}$, \\ KEISUKE TSUCHIDA ${ }^{1}$, NOBUTAKA MIZOGUCHI ${ }^{1}$, ITSUKO SERIZAWA ${ }^{1}$, HIROYUKI KATOH $^{1}$, \\ KOH IMURA $^{2}$, YOSHIKI TAKAYAMA ${ }^{2}$, SHINICHI MINOHARA ${ }^{2}$ and TADASHI KAMADA ${ }^{1}$ \\ ${ }^{1}$ Department of Radiation Oncology, Kanagawa Cancer Center, Yokohama, Japan; \\ ${ }^{2}$ Section of Medical Physics and Engineering, Kanagawa Cancer Center, Yokohama, Japan
}

\begin{abstract}
Background/Aim: The present study aimed to compare the radiation dose distribution of carbon-ion radiotherapy (CIRT) for stage I esophageal cancer with three-dimensional conformal radiotherapy (3DCRT) and volumetric modulated arc therapy (VMAT). Patients and Methods: Fifteen patients with cTlbNOMO esophageal cancer who received 3DCRT at Kanagawa Cancer Center between January 2014 and April 2019 were enrolled. The dose-volume histogram parameters of the target volume and normal organs planned with CIRT, 3DCRT, and VMAT were evaluated. Results: The homogeneity index for the target volume of CIRT was significantly lower than that of $3 D C R T$ and VMAT. In addition, the radiation dose of CIRT to the heart, lungs, spinal cord, and skin was significantly lower than that of 3DCRT and VMAT. Conclusion: Favorable dose distributions with CIRT were demonstrated compared with 3DCRT and VMAT for esophageal cancer.
\end{abstract}

One of the radical treatments for stage I esophageal cancer is radiotherapy (1). Favorable clinical outcomes have been reported for radiotherapy for esophageal cancer (1). However, treatment-related late toxicities have been recently reported when long-term survival was achieved through radiotherapy (2). Late toxicities to the heart and lungs following radiotherapy could be particularly problematic and could even be fatal (2-4). Various technologies of

This article is freely accessible online.

Correspondence to: Yosuke Takakusagi, MD, Ph.D., Department of Radiation Oncology, Kanagawa Cancer Center, 241-8515, Asahiku, Yokohama, Kanagawa, Japan. Tel: +81 455202222, Fax: +81 455202202, e-mail: y-takakusagi@kcch.jp

Key Words: Carbon-ion radiotherapy, dosimetric comparison, esophageal cancer. radiotherapy, such as intensity-modulated radiotherapy (IMRT) and particle beam radiotherapy, have been developed to reduce the radiation dose to the normal organs.

Carbon-ion radiotherapy (CIRT) was developed in 1994 by the National Institute of Radiological Sciences (Chiba, Japan) (5). CIRT has significant more physical and biological advantages compared to conventional radiation therapy using X-rays (XRT). CIRT exhibits higher radiation dose concentration and better dose distribution due to the Bragg peak and sharp penumbra, with regards to the physical aspects (6). Furthermore, with regard to the biological characteristics, the biological effect of CIRT is approximately three-fold higher than that of XRT $(7,8)$. These characteristics offer a good therapeutic outcome without severe adverse events in normal tissue. In fact, favorable clinical outcomes with CIRT have been reported for various diseases (9-11).

At Kanagawa Cancer Center (KCC), CIRT was initiated in 2015 (12). CIRT was administered using the raster scanning method in all cases at KCC. The raster scanning method involves scanning with a pencil beam of $\sim 3 \mathrm{~mm}$ in diameter, at high speed. This method, coupled with the high speed irradiates the target in a three-dimensional manner and enables radiation dose distribution with greater concentration compared to the conventional broad beam method (13). Favorable clinical outcomes have been reported with CIRT for prostate cancer at KCC (14).

Proton beam therapy (PBT) has a similar Bragg peak as CIRT, resulting in high radiation dose concentration (15). For esophageal cancer, PBT has been found to show better radiation dose distribution compared to XRT (15-17). However, no studies have compared the radiation dose distribution of CIRT and that of XRT. Therefore, in the present study, quantitative analysis was conducted to compare the radiation dose distribution of CIRT for stage I esophageal cancer with three-dimensional conformal radiotherapy (3DCRT) and volumetric modulated arc therapy (VMAT). 
A

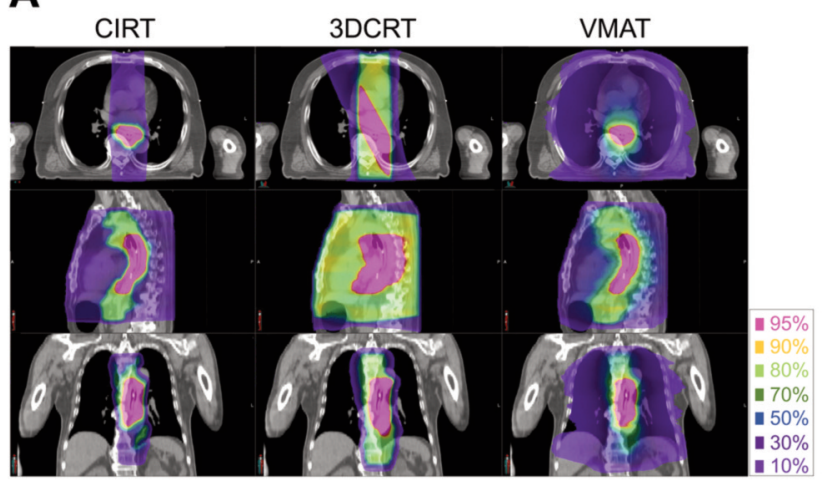

B

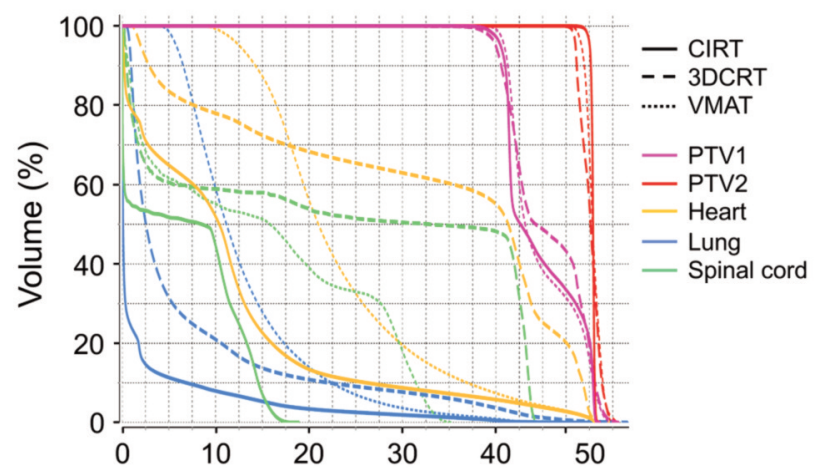

Figure 1. Comparison of a typical radiation dose distribution. For PTV1, 41.4 Gy or Gy (RBE) was administered. For PTV2, a total dose of 50.4 Gy or Gy (RBE) was administered. (a) Radiation dose distribution of CIRT, 3DCRT, and VMAT. (b) DVH of CIRT, 3DCRT, and VMAT.

\section{Patients and Methods}

Patients. The hospital institutional review board approved this study (approval number: 2020-71). The subject sample included patients with cT1bN0M0 esophageal cancer who received radiotherapy delivered by 3DCRT between January 2014 and April 2019 at KCC. From the most recent cases, we included five cases of the upper thoracic area (UT), midthoracic area (MT), and lower thoracic area (LT) for a total of 15 cases as the tumor site affects the radiation dose distribution on normal tissue $(15,18)$.

Treatment planning. Computed tomography (CT) scans were performed in 2.5-5 mm slices with free breathing. Gross tumor volume (GTV) was identified using a clip that was placed endoscopically in advance. Clinical target volume (CTV) 1 included the lymph node regions by tumor location as follows: UT esophageal cancer included the supraclavicular and superior mediastinal lymph node regions, MT esophageal cancer included the superior and inferior mediastinal lymph node regions and intra-abdominal lymph node regions, and LT esophageal cancer included the inferior mediastinal and intra-abdominal lymph node regions. CTV2 extended $2 \mathrm{~cm}$ in each longitudinal direction of the esophagus from the GTV. Planning target volume (PTV) 1 and 2 were CTV 1 and $2+5 \mathrm{~mm}$ in all directions, respectively. The heart, lungs, spinal cord, and skin were extracted as organs at risk (OAR). OAR was extracted according to the RTOG1106 OAR atlas (19). The radiation dose for the skin was evaluated upon creating an inner ring structure of 0.2 $\mathrm{mm}$ in thickness from the auto-extracted skin (20). The same structure was used for CIRT, 3DCRT, and VMAT planning.

The dose of CIRT is expressed as Gy [relative biological effectiveness (RBE)], which is defined as the physical dose multipled by the RBE of the carbon ions. The prescribed radiation dose was 41.4 Gy in XRT or Gy (RBE) in CIRT for PTV1 and an additional 9.0 Gy or Gy (RBE) for PTV2. The total dose for PTV2 was 50.4 Gy or Gy (RBE). In 3DCRT, an anteroposterior opposing portal beam was set at 41.4 Gy, and the spinal cord was shielded by irradiating at $9.0 \mathrm{~Gy}$ with an oblique opposing beam. The same beam placement was used for CIRT. For 3DCRT, the radiation dose was prescribed to the isocenter, and a plan was created to cover the CTV with $95 \%$ of the radiation dose. For VMAT, the radiation dose was prescribed to be the mean radiation dose (Dmean) of PTV2. CIRT treatment plan was created to cover $95 \%$ of the PTV with $95 \%$ of the prescription dose. The dose constraints for OARs were as follows: The maximum dose (Dmax) was 45 Gy or Gy (RBE) for the spinal cord, and V $20<20 \%$ for the lungs. Treatment plans were created with the Monaco version 5.11 (Elekta AB, Stockholm, Sweden) for 3DCRT and VMAT, and treatment plans for CIRT were created with the Monaco version 5.20 for carbon-ion scanning (Elekta AB). All treatment plans were transferred to the MIM maestro software version 6.9 (MIM Software Inc., Cleveland, $\mathrm{OH}$, USA), and dose-volume histograms (DVH) were created. The following DVH parameters were assessed: the dose covering $98 \%$ of the target volume (D98), D95, D50, D2, and the homogeneity index (HI) for PTVs. HI was calculated by (D2-D98)/D50 (21). V10-50 and D mean for the heart, with V5-V50 and Dmean for the lungs, Dmax for the spinal cord, and Dmax for the skin were also evaluated.

Statistical analysis. The DVH parameters of each treatment method were compared using the Wilcoxon matched-pairs test.

For CIRT, DVH parameters were compared according to each tumor site using Mann-Whitney $U$-test. A $p$-value of $<0.05$ was considered statistically significant. Statistical analyses were conducted using STATA software (version 13.1, College Station, TX, USA).

\section{Results}

PTV. Typical radiation dose distribution and DVH are presented in Figure 1. The DVH parameters for PTVs are summarized in Table I. There was no significant difference in D50 of PTV2 with CIRT compared with 3DCRT and VMAT ( $p=0.477,0.172$ ). In CIRT, the D98 of PTV2 was significantly higher than that in 3DCRT and VMAT, whereas D2 was significantly lower. The HI was $0.02 \pm 0.01$, $0.07 \pm 0.02$, and $0.07 \pm 0.02$ in CIRT, 3DCRT, and VMAT, respectively. HI of CIRT was significantly lower than that of 3DCRT and VMAT ( $p<0.001$ and $p<0.001$, respectively).

OARs. The DVH parameters for OARs are presented in Table II. All the CIRT domestic variables regarding the heart 
Table I. Dosimetric comparison for PTVs between 3DCRT and CIRT, VMAT and CIRT.

\begin{tabular}{|c|c|c|c|c|c|}
\hline \multirow[b]{2}{*}{ DVH parameters } & \multicolumn{3}{|c|}{ Mean \pm SD } & \multicolumn{2}{|c|}{$p$-Value } \\
\hline & CIRT & 3DCRT & VMAT & vs. 3DCRT & vs. VMAT \\
\hline \multicolumn{6}{|l|}{ PTV1 } \\
\hline D98 [Gy, Gy(RBE)] & $40.1 \pm 0.4$ & $40.9 \pm 1.4$ & $41.0 \pm 0.4$ & 0.002 & $<0.001$ \\
\hline D95 [Gy, Gy(RBE)] & $40.9 \pm 0.3$ & $41.7 \pm 1.0$ & $41.4 \pm 0.4$ & 0.007 & $<0.001$ \\
\hline D50 [Gy, Gy(RBE)] & $46.4 \pm 7.2$ & $50.0 \pm 2.9$ & $46.5 \pm 2.4$ & $<0.001$ & 0.955 \\
\hline D2 [Gy, Gy(RBE)] & $50.6 \pm 0.1$ & $52.1 \pm 0.3$ & $51.6 \pm 0.4$ & $<0.001$ & $<0.001$ \\
\hline Homogenity index & $0.23 \pm 0.07$ & $0.26 \pm 0.04$ & $0.23 \pm 0.01$ & 0.013 & 0.650 \\
\hline \multicolumn{6}{|l|}{ PTV2 } \\
\hline D98 [Gy, Gy(RBE)] & $49.7 \pm 0.3$ & $48.5 \pm 0.9$ & $48.4 \pm 0.4$ & $<0.001$ & $<0.001$ \\
\hline D95 [Gy, Gy(RBE)] & $50.0 \pm 0.1$ & $49.1 \pm 0.8$ & $48.8 \pm 0.3$ & $<0.001$ & $<0.001$ \\
\hline D50 [Gy, Gy(RBE)] & $50.4 \pm 0.1$ & $50.4 \pm 0.3$ & $50.4 \pm 0.1$ & 0.477 & 0.172 \\
\hline D2 [Gy, Gy(RBE)] & $50.7 \pm 0.1$ & $51.7 \pm 0.3$ & $52.2 \pm 0.4$ & $<0.001$ & $<0.001$ \\
\hline Homogenity index & $0.02 \pm 0.01$ & $0.07 \pm 0.02$ & $0.07 \pm 0.02$ & $<0.001$ & $<0.001$ \\
\hline
\end{tabular}

PTV: Planning target volume; 3DCRT: three-dimentional comformal radiotherapy; VMAT: volumetric modulated arc therapy; CIRT: carbon-ion radiotherapy; RBE: relative biological effectivenes; DVH: dose-volume histogram; SD: standard deviation.

Table II. Dosimetric comparison for OARs between 3DCRT and CIRT, VMAT and CIRT.

\begin{tabular}{|c|c|c|c|c|c|}
\hline \multirow[b]{2}{*}{ DVH parameters } & \multicolumn{3}{|c|}{ Mean \pm SD } & \multicolumn{2}{|c|}{$p$-Value } \\
\hline & CIRT & 3DCRT & VMAT & vs. 3DCRT & vs. VMAT \\
\hline \multicolumn{6}{|l|}{ Heart } \\
\hline V10 (\%) & $46.5 \pm 21.0$ & $69.2 \pm 28.1$ & $99.6 \pm 39.6$ & $<0.001$ & $<0.001$ \\
\hline V20 (\%) & $9.8 \pm 5.6$ & $61.4 \pm 25.8$ & $45.0 \pm 24.1$ & $<0.001$ & $<0.001$ \\
\hline V30 (\%) & $6.7 \pm 3.7$ & $57.3 \pm 24.8$ & $18.0 \pm 9.6$ & $<0.001$ & $<0.001$ \\
\hline V40 (\%) & $4.8 \pm 2.6$ & $51.1 \pm 23.5$ & $7.3 \pm 5.0$ & 0.001 & $<0.001$ \\
\hline V50 (\%) & $0.9 \pm 0.6$ & $8.3 \pm 6.6$ & $1.1 \pm 1.0$ & 0.006 & 0.068 \\
\hline Dmean $[\mathrm{Gy}, \mathrm{Gy}(\mathrm{RBE})]$ & $9.6 \pm 4.5$ & $29.1 \pm 11.7$ & $22.3 \pm 9.0$ & $<0.001$ & $<0.001$ \\
\hline \multicolumn{6}{|l|}{ Lung } \\
\hline V5 (\%) & $8.4 \pm 3.7$ & $21.6 \pm 8.3$ & $77.7 \pm 17.8$ & $<0.001$ & $<0.001$ \\
\hline V10 (\%) & $6.7 \pm 3.2$ & $14.8 \pm 5.5$ & $52.5 \pm 12.1$ & $<0.001$ & $<0.001$ \\
\hline V20 (\%) & $2.6 \pm 1.7$ & $7.7 \pm 3.3$ & $14.8 \pm 5.5$ & $<0.001$ & $<0.001$ \\
\hline V30 (\%) & $1.4 \pm 1.0$ & $5.5 \pm 2.5$ & $3.9 \pm 2.3$ & $<0.001$ & $<0.001$ \\
\hline V40 (\%) & $0.3 \pm 0.5$ & $2.2 \pm 1.2$ & $1.0 \pm 0.9$ & $<0.001$ & 0.001 \\
\hline V50 (\%) & $0.0 \pm 0.1$ & $0.1 \pm 0.2$ & $0.0 \pm 0.1$ & 0.006 & 0.157 \\
\hline Dmean [Gy, Gy(RBE)] & $1.8 \pm 0.9$ & $5.3 \pm 1.8$ & $11.4 \pm 2.3$ & $<0.001$ & $<0.001$ \\
\hline \multicolumn{6}{|l|}{ Spinal cord } \\
\hline Dmax [Gy, Gy(RBE)] & $25.6 \pm 3.5$ & $44.6 \pm 0.6$ & $41.3 \pm 2.9$ & $<0.001$ & $<0.001$ \\
\hline \multicolumn{6}{|l|}{ Skin } \\
\hline $\operatorname{Dmax}[\mathrm{Gy}, \mathrm{Gy}(\mathrm{RBE})]$ & $15.1 \pm 9.5$ & $30.1 \pm 4.6$ & $24.2 \pm 10.3$ & 0.001 & $<0.001$ \\
\hline
\end{tabular}

OAR: Organ at risk; 3DCRT: three-dimentional comformal radiotherapy; VMAT: volumetric modulated arc therapy; CIRT: carbon-ion radiotherapy; RBE: relative biological effectiveness; DVH: dose-volume histogram; SD: standard deviation.

dose were significantly lower than those of the 3DCRT plans. All the CIRT domestic variables regarding the heart dose, except for the V50, were significantly lower than those of the VMAT plans. The Dmean of the heart was $9.6 \pm 4.5$ Gy (RBE) for CIRT compared with $29.1 \pm 11.7$ Gy for 3DCRT and $22.3 \pm 9.0$ Gy for VMAT, indicating a significant difference $(p<0.001$ and $p<0.001$, respectively). All the CIRT domestic variables regarding the lung dose were significantly lower than those of the 3DCRT plans. All the CIRT domestic variables regarding the lung dose except for the V50 were significantly lower than those of the VMAT plans. The Dmean of the lungs was $1.8 \pm 0.9$ Gy (RBE) for CIRT, as compared to $5.3 \pm 1.8 \mathrm{~Gy}$ in 3DCRT, and $11.4 \pm 2.3 \mathrm{~Gy}$ in VMAT, indicating a significant difference $(p<0.001$ and 
Table III. Dosimetric comparison for OARs according to the tumor site in CIRT.

\begin{tabular}{|c|c|c|c|c|c|c|}
\hline \multirow[b]{2}{*}{ DVH parameters } & \multicolumn{3}{|c|}{ Mean \pm SD } & \multicolumn{3}{|c|}{$p$-Value } \\
\hline & UT & MT & LT & UT vs. MT & UT vs. LT & MT $v s . \mathrm{LT}$ \\
\hline \multicolumn{7}{|l|}{ Heart } \\
\hline V10 (\%) & $4.6 \pm 6.8$ & $48.7 \pm 4.1$ & $50.0 \pm 7.6$ & 0.009 & 0.009 & 0.602 \\
\hline V20 (\%) & $1.2 \pm 2.0$ & $12.2 \pm 2.3$ & $13.9 \pm 2.9$ & 0.005 & 0.009 & 0.117 \\
\hline V30 (\%) & $0.7 \pm 1.3$ & $7.6 \pm 1.6$ & $8.8 \pm 1.9$ & 0.005 & 0.009 & 0.076 \\
\hline V40 (\%) & $0.3 \pm 0.9$ & $4.9 \pm 1.4$ & $6.0 \pm 1.2$ & 0.004 & 0.009 & 0.075 \\
\hline V50 (\%) & $0 \pm 0.2$ & $1.0 \pm 0.4$ & $1.3 \pm 0.3$ & 0.006 & 0.008 & 0.059 \\
\hline Dmean $[\mathrm{Gy}(\mathrm{RBE})]$ & $1.3 \pm 1.6$ & $10.9 \pm 1.2$ & $11.1 \pm 1.7$ & 0.008 & 0.009 & 0.465 \\
\hline \multicolumn{7}{|l|}{ Lung } \\
\hline V5 (\%) & $9.8 \pm 2.7$ & $10.0 \pm 2.1$ & $3.9 \pm 2.2$ & 0.754 & 0.005 & 0.016 \\
\hline V10 (\%) & $9.4 \pm 2.0$ & $7.7 \pm 1.8$ & $1.9 \pm 1.5$ & 0.467 & 0.009 & 0.016 \\
\hline V20 (\%) & $3.1 \pm 1.0$ & $3.4 \pm 1.0$ & $0.1 \pm 0.3$ & 0.6 & 0.009 & 0.009 \\
\hline V30 (\%) & $1.7 \pm 0.7$ & $2.0 \pm 0.7$ & $0 \pm 0.2$ & 0.675 & 0.007 & 0.007 \\
\hline $\mathrm{V} 40(\%)$ & $0.6 \pm 0.5$ & $0.6 \pm 0.4$ & $0 \pm 0.1$ & 0.753 & 0.007 & 0.01 \\
\hline V50 (\%) & $0 \pm 0.1$ & $0 \pm 0.0$ & $0 \pm 0.0$ & 0.317 & 0.317 & NA \\
\hline Dmean $[\mathrm{Gy}(\mathrm{RBE})]$ & $1.9 \pm 0.6$ & $2.2 \pm 0.5$ & $0.6 \pm 0.4$ & 0.675 & 0.009 & 0.009 \\
\hline \multicolumn{7}{|l|}{ Spinal cord } \\
\hline $\operatorname{Dmax}[\mathrm{Gy}(\mathrm{RBE})]$ & $28.4 \pm 2.1$ & $25.1 \pm 3.2$ & $25.0 \pm 3.7$ & 0.028 & 0.009 & 0.602 \\
\hline \multicolumn{7}{|l|}{ Skin } \\
\hline $\operatorname{Dmax}[\mathrm{Gy}(\mathrm{RBE})]$ & $21.3 \pm 12.1$ & $15.1 \pm 4.5$ & $13.4 \pm 1.1$ & 0.047 & 0.009 & 0.117 \\
\hline
\end{tabular}

OAR: Organ at risk; CIRT: carbon-ion radiotherapy; UT: upper thorasic; MT: midthoracic; LT: lower thoracic; RBE: relative biological effectiveness; DVH: dose-volume histogram; SD: standard deviation.

$<0.001$, respectively). The Dmax of the spinal cord was significantly lower in the CIRT plans than that of the 3DCRT and VMAT plans $(p<0.001$ and $p<0.001$, respectively). The Dmax of the skin was significantly lower in the CIRT plans than that of the 3DCRT and VMAT plans $(p=0.001$ and $p<0.001$, respectively).

The DVH parameters for OARs according to the tumor site in CIRT are presented in Table III. For the heart, all parameters were significantly lower in the UT area. There was no significant difference between the MT and LT areas for DVH parameters of the heart. The Dmean of the heart was $1.3 \pm 1.6$ Gy (RBE), 10.9 \pm 12 Gy (RBE), and $11.1 \pm 1.7$ Gy (RBE) in the UT, MT, and LT areas, respectively. All the CIRT domestic variables in the LT area regarding the lung dose, except for the V50, were significantly lower than those of the MT and UT areas. There was no significant difference between the UT and MT areas for DVH parameters of the lungs. The Dmean of the lungs was 1.9 $\pm 0.6 \mathrm{~Gy}(\mathrm{RBE}), 2.2 \pm 0.5 \mathrm{~Gy}(\mathrm{RBE})$, and $0.6 \pm 0.4$ Gy (RBE) in the UT, MT, and LT areas, respectively. For both the spinal cord and the skin, the Dmax was significantly higher in the UT area than that of the MT and LT areas.

\section{Discussion}

In the present study, we compared the radiation dose distribution of CIRT, 3DCRT, and VMAT for stage I esophageal cancer. The present study demonstrated that CIRT resulted in more optimal dose distribution to the target volume and a lower dose to OARs than 3DCRT and VMAT. To the best of our knowledge, this is the first report on the comparison of the radiation dose distribution between CIRT and XRT.

HI was lower in the CIRT plans in this study. A low HI means that there is little difference in the high irradiation area and low irradiation area within the target volume, demonstrating that a uniform dose was administered (22). In XRT, when the target volume was located close to the lungs, differences in the electron density can reduce the radiation dose in the target volume. Therefore, it can be difficult to maintain dose homogeneity in the target volume treated by XRT. However, it was suggested that even for such lesions of the chest, a uniform dose could be administered with CIRT.

In radiotherapy of esophageal cancer, it is essential to reduce toxicity in the heart and lungs because late cardiopulmonary toxicities can be life-threatening (2-4). It has been reported that late toxicity to the heart and lungs is affected by excessive radiation doses to these normal organs. In the heart, V30 has been reported to be a prognostic factor of pericardial effusion (23). Shirai et al. have suggested that V40 of the heart was associated with pleural effusion (18). In addition, in another study, it was suggested that V30 of the heart was a prognostic factor of survival (24). In the lungs, V20 was considered as a risk factor for radiation pneumonitis after radiotherapy for esophageal cancer (25). It has been reported that the radiation dose to the heart was reduced by 
using IMRT (26). Because the particle beam with a Bragg peak can be reduced in organs that are deeper than the target, particle beam radiotherapy can further reduce the dose to normal organs. In fact, several studies have indicated that the radiation dose in the lungs and heart was reduced with PBT (15-17). Similar to these reports of PBT, the present study revealed that CIRT can provide significantly lower dose to the heart and lungs than XRT. CIRT reduced the mean radiation dose in the heart to $<1 / 3$ of 3DCRT and $<1 / 2$ of VMAT. The mean radiation dose to the lungs was also reduced to $1 / 3$ of 3DCRT and 1/6 of VMAT. A comparison of the heart and lungs of the CIRT group with the VMAT group indicated that there was no significant difference in terms of V50. With VMAT, a radiation dose conforming to the target volume can be administered, and it was suggested that the volume of normal organ irradiated by the prescribed dose was small.

It has also been demonstrated that PBT can reduce the dose to the spinal cord as well (16). In the present study, a similar tendency was found; CIRT reduced the dose to the spinal cord to $50 \%$ of 3DCRT and $60 \%$ of VMAT. Spinal cord disorders result in a significant reduction in the quality of life. CIRT can be used to provide treatment with greater safety.

There have been no reports to date that compared the radiation dose in the skin. In the present study, CIRT demonstrated significant lower dose distribution to the skin than 3DCRT and VMAT. It has been found that the area irradiated with $40 \mathrm{~Gy}$ (RBE) of CIRT was a prognostic factor of acute dermatitis (20). Another study demonstrated that the area irradiated with $60 \mathrm{~Gy}$ (RBE) was a predictive factor of late skin toxicity (27). In the present study, the maximum dose of CIRT was sufficiently lower than that in these reports. It was suggested that there is little risk of severe adverse skin events.

A difference in radiation dose in OARs according to the tumor location has been reported. Shirai et al. have reported a high radiation dose in the heart with lesions of the distal esophagus (18). A dosimetric comparison study of PBT has also demonstrated that the mean dose of the heart has been affected by tumor site (15). In the present study, the radiation dose to the heart was significantly low in the MT and UT areas. Concerning the lungs, the radiation dose was significantly lower at LT esophagus compared with other sites. It was considered that the radiation dose to the lungs may have been relatively low because the heart was in the beam path. Whereas, the radiation dose in UT esophagus was significantly higher for the spinal cord and skin. In UT esophagus, it was considered that the spinal cord and skin were relatively close to the target volume compared with the other sites.

This study had several limitations. The subject sample size was small, appropriate irradiation field in CIRT remained unclear, and respiratory movement was not included. In actual treatment, respiratory movement is required to be evaluated using four-dimensional CT. Furthermore, our hospital is equipped with an in-room $\mathrm{CT}$ in the CIRT treatment room. The dose distribution robustness using the in-room CT should be evaluated.

In this study, favorable dose distribution was demonstrated with CIRT compared with XRT for esophageal cancer. It is expected that reducing the radiation dose to OARs will be useful for lowering the toxicity.

\section{Conflicts of Interest}

Dr. Hiroyuki Katoh, Dr. Daisaku Yoshida and Dr. Shinichi Minohara receive research funding from Toshiba Energy Systems and Solutions Corporation.

\section{Authors' Contributions}

Y Takakusagi collected and analyzed the data and drafted the manuscript. DY, HK and TK analyzed the data and contributed to the final draft of the manuscript. KK, WA and KT collected and analyzed the data of XRT. YK, KI, Y Takayama and SM collected and analyzed the data of CIRT. NM and IS aided in writing the manuscript and contributed to the final draft of the manuscript. All Authors read and approved the final manuscript.

\section{References}

1 Kato H, Sato A, Fukuda H, Kagami Y, Udagawa H, Togo A, Ando N, Tanaka O, Shinoda M, Yamana H and Ishikura S: A phase II trial of chemoradiotherapy for stage I esophageal squamous cell carcinoma: Japan Clinical Oncology Group Study (JCOG9708). Jpn J Clin Oncol 39(10): 638-643, 2009. PMID: 19549720. DOI: 10.1093/jjco/hyp069

2 Ishikura S, Nihei K, Ohtsu A, Boku N, Hironaka S, Mera K, Muto M, Ogino T and Yoshida S: Long-term toxicity after definitive chemoradiotherapy for squamous cell carcinoma of the thoracic esophagus. J Clin Oncol 21(14): 2697-2702, 2003. PMID: 12860946. DOI: 10.1200/JCO.2003.03.055

3 Minsky BD, Pajak TF, Ginsberg RJ, Pisansky TM, Martenson J, Komaki R, Okawara G, Rosenthal SA and Kelsen DP: INT 0123 (Radiation Therapy Oncology Group 94-05) phase III trial of combined-modality therapy for esophageal cancer: high-dose versus standard-dose radiation therapy. J Clin Oncol 20: 11671174, 2002. PMID: 11870157. DOI: 10.1200/JCO.2002.20.5.1167

4 Morota M, Gomi K, Kozuka T, Chin K, Matsuura M, Oguchi M, Ito $\mathrm{H}$ and Yamashita $\mathrm{T}$ : Late toxicity after definitive concurrent chemoradiotherapy for thoracic esophageal carcinoma. Int $\mathbf{J}$ Radiat Oncol Biol Phys 75: 122-128, 2009. PMID: 19327900. DOI: $10.1016 /$ j.ijrobp.2008.10.075

5 Akakura K, Tsujii H, Morita S, Tsuji H, Yagishita T, Isaka S, Ito H, Akaza H, Hata M, Fujime M, Harada M and Shimazaki J: Phase I/II clinical trials of carbon ion therapy for prostate cancer. Prostate 58(3): 252-258, 2004. PMID: 14743464. DOI: 10.1002/pros. 10328

6 Schulz-Ertner D and Tsujii H: Particle radiation therapy using proton and heavier ion beams. J Clin Oncol 25: 953-964, 2007. PMID: 17350944. DOI: 10.1200/JCO.2006.09.7816

7 Kanai T, Endo M, Minohara S, Miyahara N, Koyama-ito H, Tomura H, Matsufuji N, Futami Y, Fukumura A, Hiraoka T, Furusawa Y, Ando K, Suzuki M, Soga F and Kawachi K: Biophysical characteristics of HIMAC clinical irradiation system for heavy-ion 
radiation therapy. Int J Radiat Oncol Biol Phys 44: 201-210, 1999. PMID: 10219815. DOI: 10.1016/s0360-3016(98)00544-6

8 Kanai T, Matsufuji N, Miyamoto T, Mizoe J, Kamada T, Tsuji H, Kato H, Baba M and Tsujii H: Examination of GyE system for HIMAC carbon therapy. Int J Radiat Oncol Biol Phys 64: 650656, 2006. PMID: 16414376. DOI: 10.1016/j.jrobp.2005.09.043

9 Nomiya T, Tsuji H, Kawamura H, Ohno T, Toyama S, Shioyama Y, Nakayama Y, Nemoto K, Tsujii $\mathrm{H}$ and Kamada T: A multiinstitutional analysis of prospective studies of carbon-ion radiotherapy for prostate cancer: a report from the Japan carbonion radiation oncology study group (J-CROS). Radiother Oncol 121(2): 288-293, 2016. PMID: 27836119. DOI: 10.1016/j. radonc.2016.10.009

10 Kawashiro S, Yamada S, Okamoto M, Ohno T, Nakano T, Shinoto M, Shioyama Y, Nemoto K, Isozaki Y, Tsuji $\mathrm{H}$ and Kamada T: Multi-institutional study of carbon-ion radiotherapy for locally advanced pancreatic cancer: Japan Carbon-ion Radiation Oncology Study Group (J-CROS) Study 1403 Pancreas. Int J Radiat Oncol Biol Phys 101(5): 1212-1221, 2018. PMID: 29907490. DOI: 10.1016/j.ijrobp.2018.04.057

11 Koto M, Demizu Y, Saitoh JI, Suefuji H, Tsuji H, Okimoto T, Ohno T, Shioyama Y, Takagi R, Nemoto K, Nakano T and Kamada T: Japan carbon-ion radiation oncology study group. Multicenter Study of Carbon-Ion Radiation Therapy for Mucosal Melanoma of the Head and Neck: Subanalysis of the Japan Carbon-Ion Radiation Oncology Study Group (J-CROS) Study (1402 HN). Int J Radiat Oncol Biol Phys 97(5): 1054-1060, 2016. PMID: 28332989. DOI: 10.1016/j.ijrobp.2016.12.028

12 Nakayama Y, Minohara S, Nonaka T, Nomiya T, Kusano Y, Takeshita E, Mizoguchi N and Hagiwara Y: The ion-beam radiation oncology Center in Kanagawa (i-ROCK) carbon ion Facility at the Kanagawa Cancer Center. Int J Part Ther Winter 2(3): 478-480, 2016. PMID: 31772959. DOI: 10.14338/IJPT-15-00024.1

13 Minohara S, Fukuda S, Kanematsu N, Takei Y, Furukawa T, Inaniwa T, Matsufuji N, Mori S and Noda K: Recent innovations in carbon-ion radiotherapy. J Radiat Res 51(4): 385-392, 2010. PMID: 20679740. DOI: 10.1269/jrr.10028

14 Takakusagi Y, Katoh H, Kano K, Anno W, Tsuchida K, Mizoguchi N, Serizawa I, Yoshida D and Kamada T: Preliminary result of carbon-ion radiotherapy using the spot scanning method for prostate cancer. Radiat Oncol 15(1): 127, 2020. PMID: 32460889. DOI: 10.1186/s13014-020-01575-7

15 Shiraishi Y, Xu C, Yang J, Komaki R and Lin SH: Dosimetric comparison to the heart and cardiac substructure in a large cohort of esophageal cancer patients treated with proton beam therapy or Intensity-modulated radiation therapy. Radiother Oncol 125(1): 4854, 2017. PMID: 28917586. DOI: 10.1016/j.radonc.2017.07.034

16 Hirano Y, Onozawa M, Hojo H, Motegi A, Zenda S, Hotta K, Moriya S, Tachibana H, Nakamura N, Kojima T and Akimoto T: Dosimetric comparison between proton beam therapy and photon radiation therapy for locally advanced esophageal squamous cell carcinoma. Radiat Oncol 13: 23, 2018. PMID: 29426342. DOI: 10.1186/s13014-018-0966-5

17 Liu C, Bhangoo RS, Sio TT, Yu NY, Shan J, Chiang JS, Ding JX, Rule WG, Korte S, Lara P, Ding X, Bues M, Hu Y, DeWees T, Ashman JB and Liu W: Dosimetric comparison of distal esophageal carcinoma plans for patients treated with small-spot intensity-modulated proton versus volumetric-modulated arc therapies. J Appl Clin Med Phys 20(7): 15-27, 2019. PMID: 31112371. DOI: $10.1002 / \mathrm{acm} 2.12623$
18 Shirai K, Tamaki Y, Kitamoto Y, Murata K, Satoh Y, Higuchi K, Nonaka T, Ishikawa $\mathrm{H}$, Katoh $\mathrm{H}$, Takahashi $\mathrm{T}$ and Nakano $\mathrm{T}$ : Dose-volume histogram parameters and clinical factors associated with pleural effusion after chemoradiotherapy in esophageal cancer patients. Int J Radiat Oncol Biol Phys 80(4): 1002-1007, 2011. PMID: 20542385. DOI: 10.1016/j.ijrobp.2010.03.046

19 Kong FM, Ritter T, Quint DJ, Senan S, Gaspar LE, Komaki RU, Hurkmans CW, Timmerman R, Bezjak A, Bradley JD, Movsas B, Marsh L, Okunieff P, Choy H and Curran WJ Jr: Consideration of dose limits for organs at risk of thoracic radiotherapy: atlas for lung, proximal ronchial tree, esophagus, spinal cord, ribs, and brachial plexus. Int J Radiat Oncol Biol Phys 81: 1442-1457, 2011. PMID: 20934273. DOI: 10.1016/j.ijrobp.2010.07.1977

20 Takakusagi Y, Saitoh JI, Kiyohara H, Oike T, Noda SE, Ohno T and Nakano T: Predictive factors of acute skin reactions to carbon ion radiotherapy for the treatment of malignant bone and soft tissue tumors. Radiat Oncol 12(1): 185, 2017. PMID: 29166945. DOI: $10.1186 / \mathrm{s} 13014-017-0927-4$

21 International Commission on Radiation Units and Measurements: Report 83. Prescribing, recording, and reporting photon-beam intensity-modulated radiation therapy (IMRT). J ICRU 10(1), 2010. DOI: 10.1093/jicru/ndq002

22 Kubo N, Saitoh JI, Shimada H, Shirai K, Kawamura H, Ohno T and Nakano T: Dosimetric comparison of carbon ion and X-ray radiotherapy for Stage IIIA non-small cell lung cancer. J Radiat Res 57(5): 548-554, 2016. PMID: 27242341. DOI: 10.1093/jrr/rrw041

23 Wei X, Liu HH, Tucker SL, Wang S, Mohan R, Cox JD, Komaki $\mathrm{R}$ and Liao Z: Risk factors for pericardial effusion in inoperable esophageal cancer patients treated with definitive chemoradiation therapy. Int J Radiat Oncol Biol Phys 70(3): 707-714, 2008. DOI: 10.1016/j.ijrobp.2007.10.056

24 Bradley JD, Paulus R, Komaki R, Masters G, Blumenschein G, Schild S, Bogart J, Hu C, Forster K, Magliocco A, Kavadi V, Garces YI, Narayan S, Iyengar P, Robinson C, Wynn RB, Koprowski C, Meng J, Beitler J, Gaur R, Curran W Jr and Choy $\mathrm{H}$ : Standard-dose versus high-dose conformal radiotherapy with concurrent and consolidation carboplatin plus paclitaxel with or without cetuximab for patients with stage IIIA or IIIB non-smallcell lung cancer (RTOG 0617): a randomised, two-by-two factorial phase 3 study. Lancet Oncol 16(2): 187-199, 2015. PMID: 25601342. DOI: 10.1016/S1470-2045(14)71207-0

25 Tonison JJ, Fischer SG, Viehrig M, Welz S, Boeke S, Zwirner K, Klumpp B, Braun LH, Zips D and Gani C: Radiation pneumonitis after intensity-modulated radiotherapy for esophageal cancer: Institutional data and a systematic review. Sci Rep 9(1): 2255, 2019. PMID: 30783157. DOI: 10.1038/s41598-018-38414-5

26 Wu Z, Xie C, Hu M, Han C, Yi J, Zhou Y, Yuan H and Jin X: Dosimetric benefits of IMRT and VMAT in the treatment of middle thoracic esophageal cancer: is the conformal radiotherapy still an alternative option? J Appl Clin Med Phys 15(3): 93-101, 2014. PMID: 24892336. DOI: 10.1120/jacmp.v15i3.4641

27 Yanagi T, Kamada T, Tsuji H, Imai R, Serizawa I and Tsujii H: Dose-volume histogram and dose-surface histogram analysis for skin reactions to carbon ion radiotherapy for bone and soft tissue sarcoma. Radiother Oncol 95(1): 60-65, 2010. PMID: 19767117. DOI: $10.1016 /$ j.radonc.2009.08.041

Received September 28, 2020

Revised October 9, 2020

Accepted October 12, 2020 